\title{
STATISTICAL EXPRESSIONS ON WATER BASED WILDFIRE SUPPRESSION IN SWEDEN, 1996-2011
}

\author{
R. HANSEN \\ Mälardalen University, Sweden.
}

\begin{abstract}
Little research has been conducted with respect to water requirements for wildfire suppression. An exploratory and robust regression analysis was conducted on statistical material extracted from a Swedish database, focusing on data related to the fire suppression. The variables included are the amount of water applied, application rate of water, extinguishing time, flame height, and the total fire area. The results of the analysis with potential relationships are described, discussed, and compared with results from earlier research. After initial outlier detection, a robust regression analysis was performed and relationships developed. It was found that the developed relationships could predict the dependent variable very well; this was especially the case with the extinguishing time as a function of the total fire area. The relationship with the lowest ability to predict the dependent variable was the application rate of water as a function of the total fire area. The large number of parameters and their included uncertainties could be an explanation to the increased inability to predict the dependent variable. When comparing the control times of earlier conducted building fire studies with the extinguishing times of developed relationships, it was found that the control times were much longer than the corresponding extinguishing times. The reason behind was most likely due to the difference in the fire area applied in the equations. The resulting relationships for the grass fuel type were found to have lower ability to predict the dependent variable. The reason behind this is unclear and needs to be investigated further.
\end{abstract}

Keywords: Extinguishing time, robust regression analysis, water application, wildfire suppression.

\section{INTRODUCTION}

The development of decision tools is an essential activity in the fire and rescue sector, e.g. for wildfire suppression. One of the desired and needed tools would be the estimation of how much water is required for extinguishing a wildfire. Little research has been conducted with respect to water requirements for wildfire suppression. Stechishen [1] and Stechishen and Little [2] used a spray rig to water uniformly across a fuel bed, developing equations relating the penetration depth of the water into the fuel bed required to extinguish the fire with a certain fire intensity. Loane and Gould [3] used experimental data from aerial drops on fires in various fuels, smallscale laboratory experiments, and field studies to develop equations to calculate the depth of water required for fire suppression as a function of fire intensity. Douglas [4] estimated a minimum water flow requirement per meter of fireline as 10-20 litres/minute for surface fires in south eastern Australian pine forests. Andrews and Rothermel [5] and Alexander [6] used flame length and fireline intensity to determine the appropriate fire suppression activities. Alexander stated that suppression using pressurized water would be an appropriate action for fireline intensities up to $2000 \mathrm{~kW} / \mathrm{m}$. Calculating the total amount of water required for extinguishing a wildfire, the duration of water application has to be accounted for and multiplied by the water application rate. In an earlier paper by Hansen [7] it was pointed out that the duration of water application - when estimating the amount of water required to extinguish wildfires - will have to be studied further as the only studies performed have been with respect to fires in buildings by Thomas [8], Baldwin [9]. Being able to estimate the amount of water required would facilitate the work on the site of the fire and present a useful decision tool. Until now no study has been performed with respect to duration of water application at a wildfire. 
In two earlier studies by Thomas [8], Baldwin [9], the required duration of the water application is presented for building fires. The study by Thomas was based on statistical data from local fire services describing 48 fires and Baldwin used data from 134 fires. The studies led to two individual equations for the time duration of the water application in order to bring the fire under control $t_{\text {control }}$ - as a function of the fire area. Thomas presented the equation:

$$
t_{\text {control }}=197 \cdot A_{f}^{0.5} \quad A_{f}>200 \mathrm{~m}^{2}
$$

where $A_{f}$ is the fire area $\left(\mathrm{m}^{2}\right)$. Baldwin presented the following equation:

$$
t_{\text {control }}=100 \cdot A_{f}^{0.559} \quad A_{f}>20 \mathrm{~m}^{2}
$$

Baldwin pointed out that factors such as the level of experience of the firefighter, applied nozzle technique, etc. will largely influence the amount of water used on a wildfire. Due to the complexity of the operational aspects a statistical approach is suggested in order to overcome this problem.

The aim of this paper besides establishing similar time duration equations applicable to wildfires is also to perform a broader study on the statistical material in order to develop additional equations applicable and useful during the suppression of wildfires. During the work on this paper statistical material from a database administered by the Swedish Civil Contingencies Agency (MSB) has been extracted, where the data from the database was contributed by the local fire services.

In the following, the database used during the study and the extracted data from the database are outlined. The methodology used as well as an ensuing exploratory analysis is then described. The results of the exploratory analysis with potential relationships are described and discussed. The results are also compared with the results of Thomas [8]; Baldwin [9]. Finally, conclusions are drawn based upon the results and the corresponding discussion.

\section{STATISTICAL MATERIAL}

Every municipality in Sweden is required by law to document every incident that leads to the intervention of the municipal fire department. The minimum level of documentation is generally fulfilled by the use of incident reports. Before 1996 every municipality had its own type of incident report, which of course made data collection and analysis difficult. In order to obtain a more systematic approach and to be able to give a national picture of the evolution of accidents, an incident report was developed - common to all fire and rescue services - during the period 19941995. After a nationwide training program, the incident report form was first used in January 1996. The period 1996-1997 should be regarded as a trial period. The data today is collected in a database at the Swedish Civil Contingencies Agency. In 2012 the database contained a total of about 1.7 million entries [10]. The incident report consists of a total of ten pages with a mandatory main part of four pages where for example the position of the incident, time of incident, resources used, etc. should be filled in and the following additional parts to be filled in depending upon the type of incident:

- Automatic alarm with no fire or gas release

- Fire in a building

- Fire not in a building (e.g. a wildfire)

- Trafic accident

- Drowning

- Release of hazardous substance 
From the database, the following parameters were extracted for the years 1996-2011 for the ensuing analysis [11]:

- Point of time of arrival at the scene of the fire.

- Point of time when water was first applied on the fire.

- Date and point in time when the fire operations on the scene were terminated. This point of time marks the end of active fire suppression activities. It will vary from fire to fire. In some cases the activities will end before the mop up of the fire has been concluded and in other cases the activities will end when the fire is completely extinguished and the mop up operations are concluded as well.

- Maximum flame height at the time of arrival (this parameter was introduced in 2005 and is thus lacking for the period 1996-2004).

- Amount of water used during the extinguishing operations.

- Total fire area.

A total of 82,303 fires were included in the material extracted from the database.

The fuel types available in the database are:

- Grass

- Forest

- Other types of vegetation

Only the grass data was found to be adequately homogeneous and specific in order to allow for investigation related to fuel specification.

\section{METHODS}

The following potential relationships were selected for analysis and study:

- The amount of water used $\left(V_{\text {water }}\right)$ as a function of the extinguishing time $(t)$.

- The amount of water used as a function of the total fire area.

- The amount of water used as a function of the flame height $\left(H_{f}\right)$.

- The application rate of water $\left(V_{\text {water }}\right)$ as a function of the total fire area.

- The application rate of water as a function of the flame height.

- The extinguishing time as a function of the total fire area.

- The extinguishing time as a function of the flame height.

In all cases the relationships were investigated for the grass fuel type as well as all fuel types emerged into one. The relationships selected were chosen as they contain an extinguishing parameter (i.e. the amount of water used, application rate, etc.) as a function of a variable that is easily recorded (i.e. the extinguishing time) or easily observed (i.e. the total fire area or flame height) at a fire.

Initially - during the analysis - all wildfires with incomplete data were erased and excluded in the ensuing analysis. When studying the statistical material it became obvious that the material contained several outliers mainly due to human errors (entering data using an incorrect unit, etc.) and outlier identification and elimination were performed during the exploratory analysis as well as during the robust regression analysis. Water suppression values that were smaller than 100 were excluded in the analysis, as it was unclear whether the value was expressed in liter or cubic meter. The amount of water was supposed to be given in cubic meter in the incident report form. But for smaller fires the same fire area would result in amounts of water ranging from one to 100 and thus 
resulting in a considerable uncertainty of the actual amount of water used. All water amounts that ended with the number one were also excluded as it was highly probable that the number one in many cases could actually be the letter "l" as in liter.

The mean value of each population and independent value was thereafter calculated, this in order to simplify the analysis as the number of wildfires included in the analysis was very large.

In order to ensure normal distribution and therefore normal means of the populations, the central theorem was applied and all populations with less than 30 samples were discarded.

During the analysis, the extinguishing time was set equal to the time interval from when water was first applied on the fire until the time the fire operations were terminated (as the time of control is not included in the statistical material). This time interval will be longer than the time interval to get the fire under control and it will in some cases also include mop up time.

For each potential relationship, the following analysis or calculations were performed after the initial outlier detection and elimination had been performed:

- Study of scatter graphs.

- Robust regression analysis with additional outlier elimination.

- Residual plot/analysis.

- Calculation of residual sum of squares (RSS).

- Calculation of the coefficient of determination $\left(\mathrm{R}^{2}\right)$.

The software that was used during the analysis and calculations were the XLfit from IDBS (applying Tukey's biweight during the robust regression analysis), SYSTAT 13 from SYSTAT software (applying least trimmed squares (LTS) during the robust regression analysis) and Microsoft Excel add-in: Analysis ToolPak (containing data analysis tools for statistical and technical analysis). The choice of the Tukey's biweight and the LTS as applicable methods is motivated below.

\section{EXPLORATORY ANALYSIS AND ROBUST REGRESSION ANALYSIS}

When studying the values for the wildfires it was found that the effect of outlier values greatly affected the mean values. Therefore further outlier detection and elimination was conducted. For every population, the standard deviation method was applied and samples that exceeded the double standard deviation were excluded in the following analysis in order to minimize the influence of outlier values and increase the robustness of the output data. The method has two disadvantages: the failure of detecting multiple outliers where extreme outlier values are involved - by increasing the standard deviation considerably - and the difficulty detecting outliers when the sample size is small - as the outlier value will have too great an impact on the average as well as the standard deviation value. The latter issue is overcome by invoking the central theorem and discarding all populations with less than 30 samples; normal sample means were assumed for the remaining populations. The former issue was overcome by using a robust regression method during the regression analysis, where additional outlier values were identified and excluded.

The initial step of the robust regression analysis consisted of visually studying the various scatterplots in order to find out what potential functions exist for the different relationships; this was in order to be able to analyze the outcome of the ensuing best fit operations. In all but two cases the scatterplots were found to most likely resemble a logarithmic function or a power function. But in the case of the amount of water used as a function of the extinguishing time or the water application rate as a function of the flame height - for both the grass fuel type as well as the general fuel type - the appearance of the scatterplot is somewhat unclear and could possibly be represented by a linear function, an exponential function or a power function. 
The next step would be to perform a linear/non-linear regression analysis, seeking relationships between independent variables and corresponding dependent variables. Merely applying least square estimates when performing a regression analysis would result in undesirable results as outlier values were found in the various scatterplots. In order to overcome the problem with outliers during the regression analysis, robust regression methods were applied in order to identify the outliers and eliminate them. The M-estimator Tukey's biweight was applied where a method which is robust to outliers in predominantly the dependent variable was desirable and the LTS was applied where a method which is robust to outliers in predominantly the independent variable was desirable. The outlier values were detected and eliminated from the data in all cases. After the elimination of the outliers, a least squares fit was performed in the cases where LTS were applied in order to find the relationships with the best fit. In the cases where Tukey's biweight were applied, a weighted least squares fit was used during the curve fitting process.

When applying Tukey's biweight, data are down-weighed depending on its distance from the median absolute value and extreme outliers are set to zero. The procedure is conducted iteratively until a convergence criterion is achieved. Tukey's biweight function of the variable $\varepsilon$ is expressed as [12]:

$$
\psi(\varepsilon)=\left\{\begin{array}{cl}
\varepsilon \cdot\left(1-\varepsilon^{2} / t_{\text {cutoff }}^{2}\right)^{2} & ,|\varepsilon|<t_{\text {cutoff }} \\
0 & ,|\varepsilon| \geq t_{\text {cutoff }}
\end{array}\right.
$$

Tukey's biweight is a redescending function that rejects data being equal or lying further than $t_{\text {cutoff }}$ from the model. The cutoff point of the function is set with care. During the use of Tukey's biweight a cutoff point of six was used when detecting outliers.

In the least trimmed squares method, the objective is to minimize the sum of the smallest $\mathrm{k} \%$ squared residuals, where $\mathrm{k}$ is a subset of the total set. The method provides a robust alternative, insensitive to outliers as long as the outliers constitute less than $\mathrm{k} \%$ of the total set.

The residual was calculated using the following expression:

$$
e=Y-\breve{Y}
$$

where $Y$ is the original data value and $\breve{Y}$ is the predicted value from the regression line.

The sum of the squared residuals in the least trimmed squares method-having a single independent variable-was calculated applying the following formula:

$$
\mathrm{RSS}=\sum_{i=1}^{n}\left(Y_{i}-\breve{Y}_{i}\right)^{2}
$$

During the use of the least trimmed squares, a cutoff point of three was used when detecting outliers. During the robust regression analysis the relationship with the highest $\mathrm{R}^{2}$ value was not always selected as the most potential relationship. This was the case when the number of outlier knockouts was large in comparison with the number of populations and therefore increasing the uncertainty of the result.

\section{RESULTS}

In order to assess the reliability of the various potential relationships, a residual analysis was performed [13], the RSS as well as the $\mathrm{R}^{2}$ values were calculated. During the residual analysis, the potential relationships were evaluated and in some cases the potential relationship was discarded as the residual analysis showed that the plot was not randomly centered at zero, indicating that the relationship was not optimal. In those cases a new robust regression analysis was performed, resulting in a new potential relationship. In the residual analysis, the smaller RSS calculated the better fit 
of the proposed model. See Table 1 for the results of the robust regression analysis, method applied for the various potential relationships, and the residual analysis.

\subsection{The amount of water used as a function of the extinguishing time}

The best fit with respect to the general fuel type:

$$
V_{\text {water }}=8.826 \cdot e^{0.0001095 \cdot t}-7.531 \quad 60 \leq t \leq 14400
$$

The most potential relationship suggests an exponential development which could be explained by the longer extinguishing times being linked with the larger and more intensive wildfires that are more difficult to extinguish and require larger amounts of water.

When studying the graph in Fig. 1 it can be seen that the data lies close to the curve suggesting that eqn (6) predicts the actual amount of water very well, which is also indicated by an $\mathrm{R}^{2}$ value of 0.91 .

The best fit with respect to the grass fuel type:

$$
V_{\text {water }}=5.333 \cdot e^{0.0001467 \cdot t}-4.283 \quad 60 \leq t \leq 5400
$$

Same as for the general fuel type equation, eqn (7) also suggests an exponential development and the same explanation as for eqn (6) could be applied.

When studying the graph in Fig. 2 it can be seen that the data is generally not positioned very close to the curve. The scatter and deviation increases with higher values. An $\mathrm{R}^{2}$ value of 0.76 enforces the conclusion that eqn (7) does not predict the actual amount of water especially well. When comparing the results using eqn (6) with the corresponding results of eqn (7), it is noted that the appearance of the two curves is similar, but the difference in value increases with increasing time. Thus, the required amount of water does not increase more rapidly for the flashy grass fuel type than for the general fuel type with increasing extinguishing time.

5.2 The amount of water used as a function of the total fire area

The best fit with respect to the general fuel type is:

$$
V_{\text {water }}=1045-\frac{1042}{1+e^{-9.66333+0.641099 \cdot \ln \left(A_{f}\right)}} \quad 1 \leq A_{f} \leq 150000
$$

The most potential relationship describes a logarithmic development, i.e. increasing rapidly for lower values and leveling off for higher values. This could be explained by the fact that the total fire area is not equal to the active fire area, the area that could be directly related to the amount of water being used. As the total fire area increases, the ratios of the active fire area to the total fire area decreases after an initial acceleration phase and thus the leveling off at higher values.

Looking at the graph in Fig. 3, it is noted that the data lies close to the curve suggesting that eqn (8) predicts the actual amount of water very well, this is further enforced by an $\mathrm{R}^{2}$ value of 0.95 .

The best fit with respect to the grass fuel type is:

$$
V_{\text {water }}=1.373 \cdot A_{f}^{0.2126}-0.4474 \quad 1 \leq A_{f} \leq 40000
$$

Same as for eqn (8), eqn (9) also describes a logarithmic development, but leveling off at much lower values. This latter observation could possibly be attributed to the flashier fuel burnt off faster and thus further reducing the ratio between the active fire area and the total fire area. 


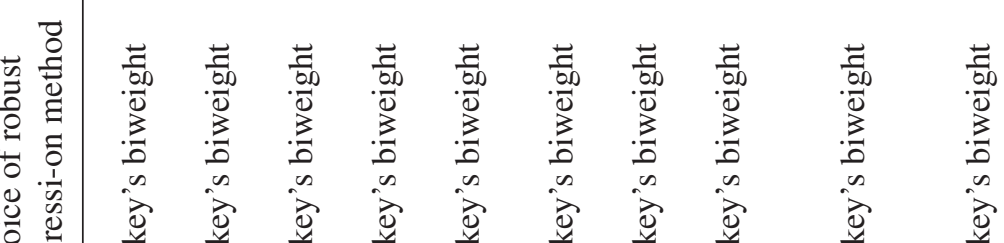

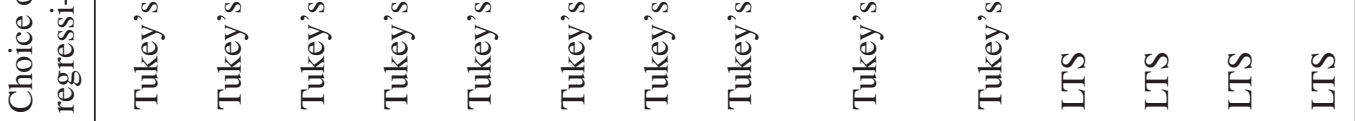

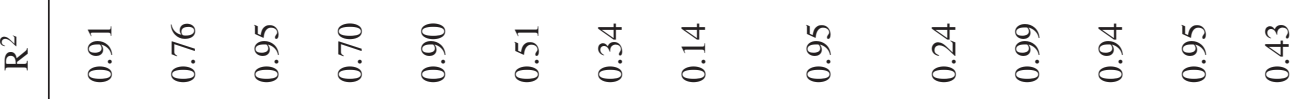

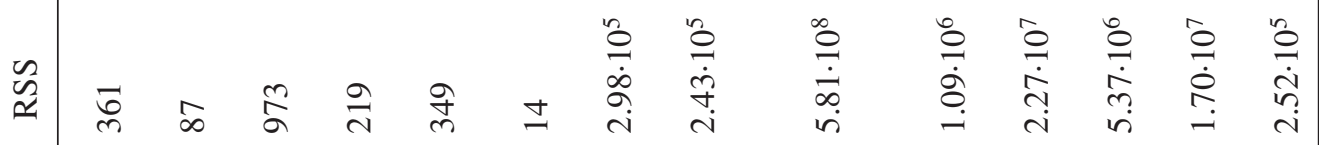

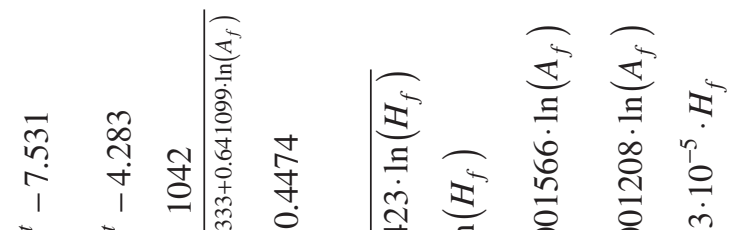

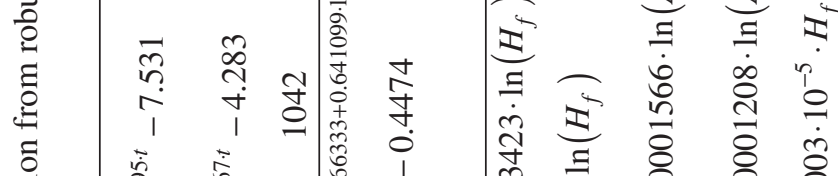

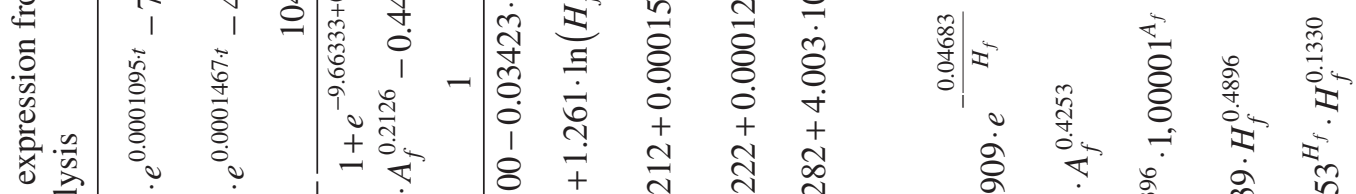

ส

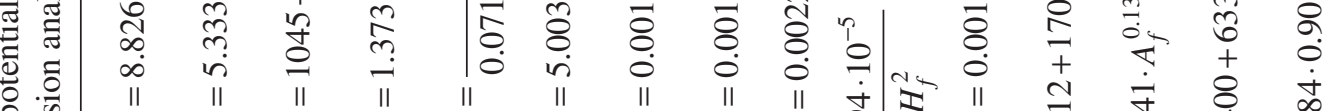

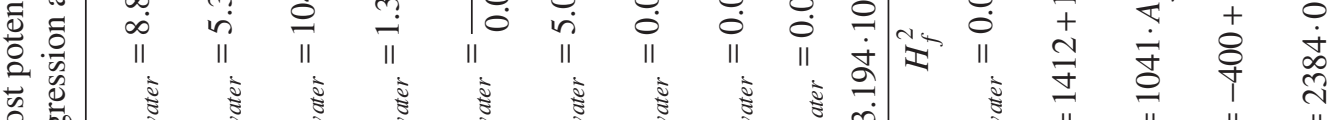

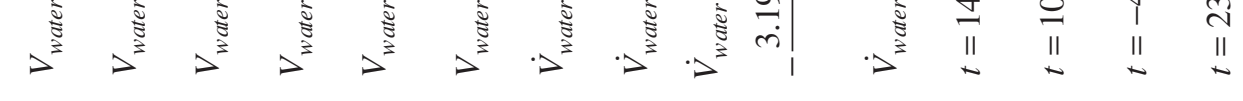

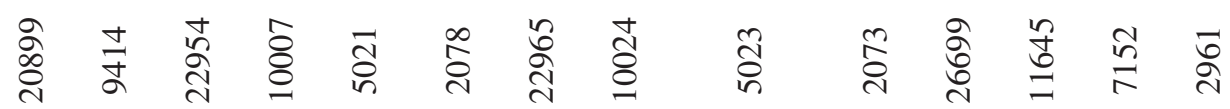

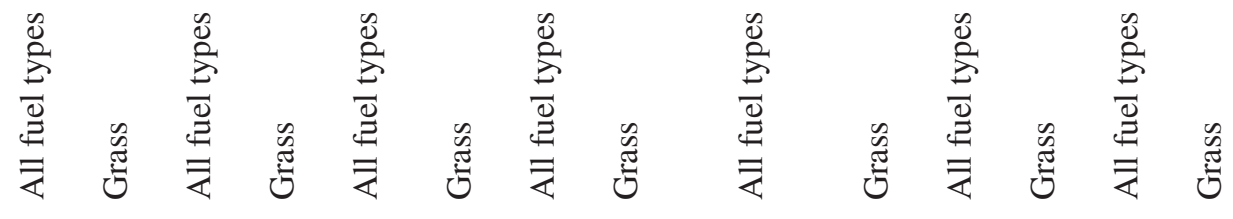

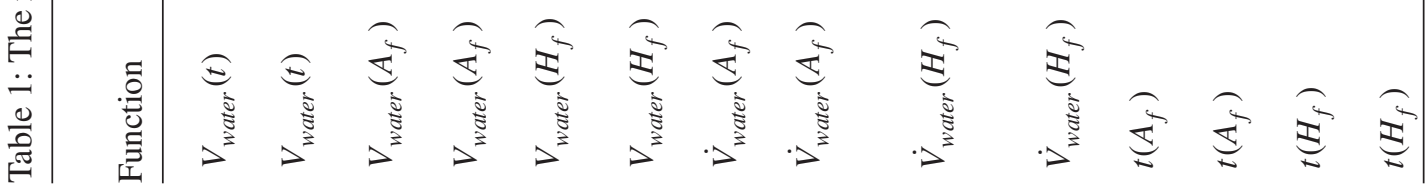




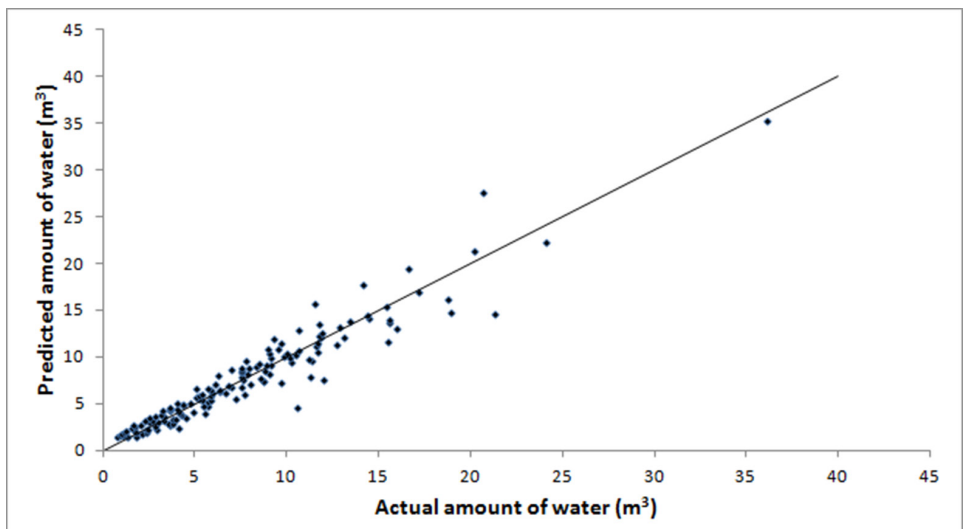

Figure 1: The amount of water used during suppression-general fuel type.

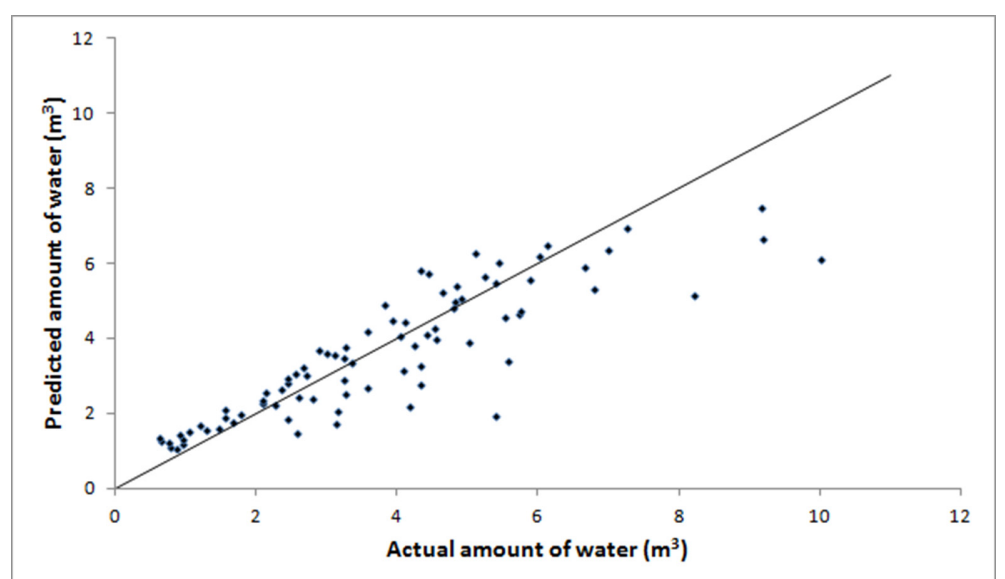

Figure 2: The amount of water used during suppression-grass fuel type.

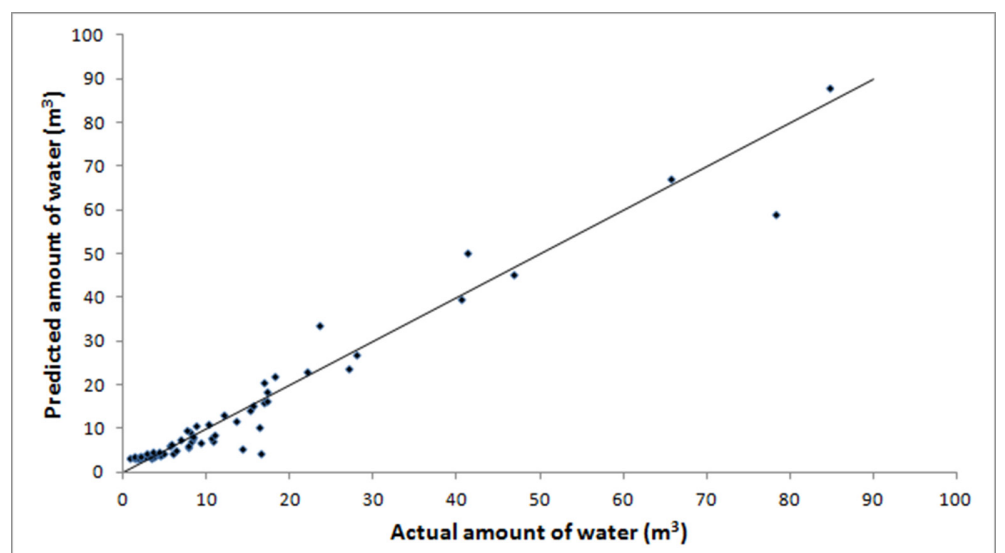

Figure 3: The amount of water used during suppression-general fuel type. 


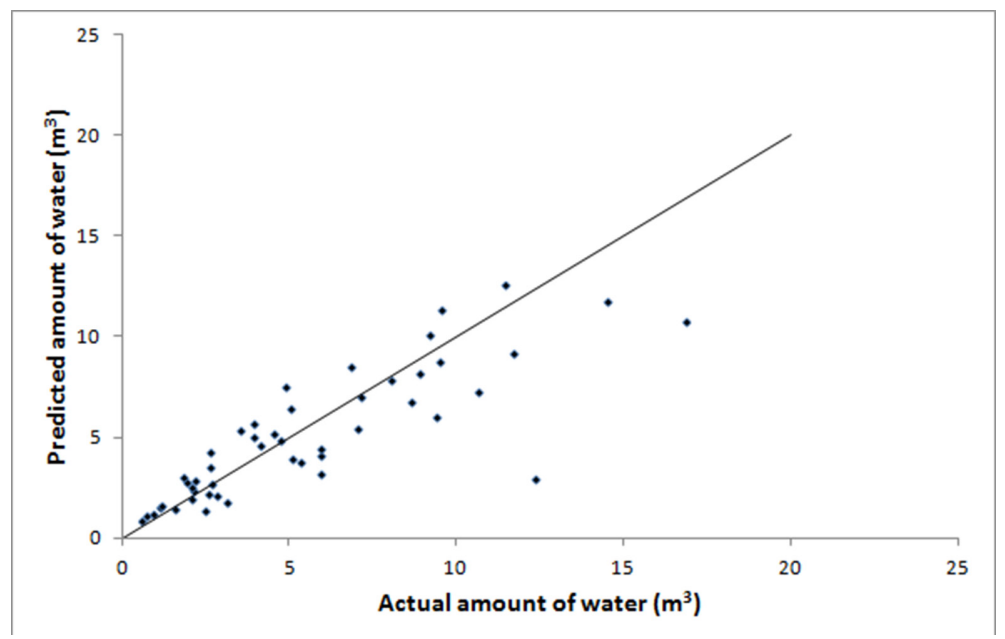

Figure 4: The amount of water used during suppression-grass fuel type.

The data points in graph in Fig. 4 are generally not positioned especially close to the curve, which indicates that eqn (9) does not predict the actual amount of water very well. An $\mathrm{R}^{2}$ value of 0.70 is in accordance with this observation.

\subsection{The amount of water used as a function of the flame height}

The best fit with respect to the general fuel type is:

$$
V_{\text {water }}=\frac{1}{0.07100-0.03423 \cdot \ln \left(H_{f}\right)} \quad 0.1 \leq H_{f} \leq 5
$$

Equation (10) has a logarithmic/linear appearance for lower values, but an exponential development for higher values (starting at approximately $H_{f}=3 \mathrm{~m}$ ). This sudden increase could possibly be explained by the very large flame heights and corresponding heat release rates requiring very large amounts of water, but also decreasing the efficiency of the water application.

An $\mathrm{R}^{2}$ value of 0.90 and data points that are close to the predicting curve in Fig. 5 suggest that eqn (10) predicts the actual amount of water very well. The limited number of data points is not due to few observations, but due to limited amount of discrete values of the flame height.

The best fit with respect to the grass fuel type is:

$$
V_{\text {water }}=5.003+1.261 \cdot \ln \left(H_{f}\right) \quad 0.1 \leq H_{f} \leq 3
$$

Equation (11) describes a logarithmic development, leveling off at low values and staying almost constant. As can be noted, eqn (11) has an upper limit of $H_{\mathrm{f}}=3 \mathrm{~m}$, which is the flame height where eqn (10) initiates an exponential development.

The data points in graph in Fig. 6 are with few exceptions not positioned very close to the curve. An $\mathrm{R}^{2}$ value of 0.51 further strengthens the conclusion that eqn (11) does not predict the actual amount of water very well. 


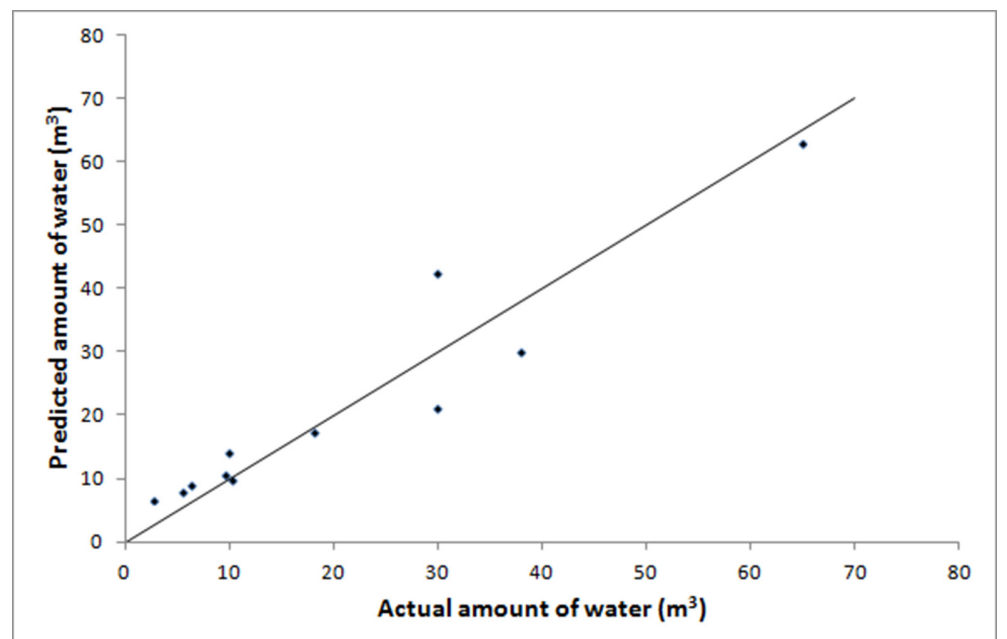

Figure 5: The amount of water used during suppression - general fuel type.

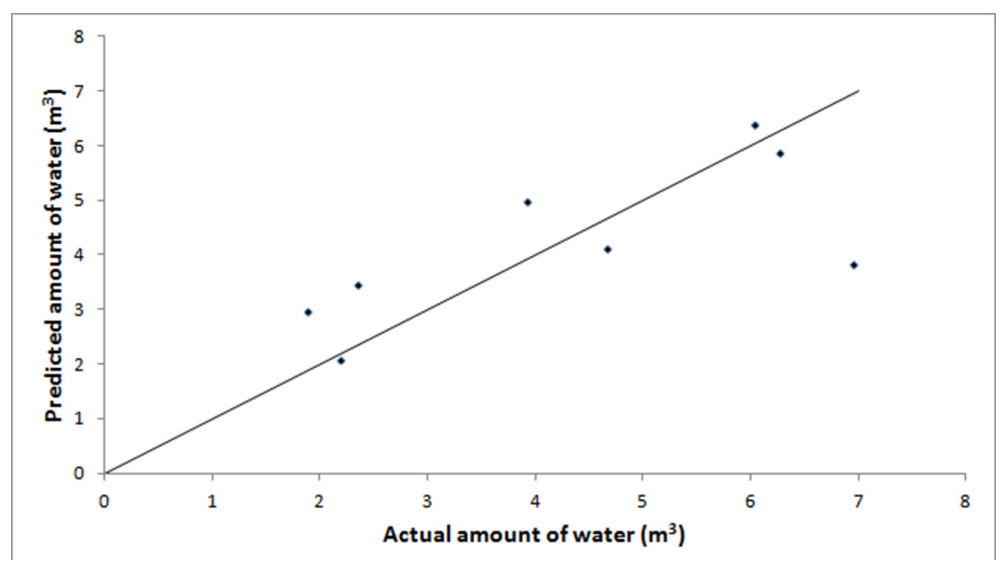

Figure 6: The amount of water used during suppression - grass fuel type.

5.4 The application rate of water as a function of the total fire area

The best fit with respect to the general fuel type is:

$$
\dot{V}_{\text {water }}=0.001212+0.0001566 \cdot \ln \left(A_{f}\right) \quad 1 \leq A_{f} \leq 150000
$$

When studying eqn (12) the same development for several of the earlier equations can be found, i.e. a logarithmic development, leveling off for higher values. This is not surprising as the earlier reasoning regarding the ratio of the active fire area to the total fire area applies here as well.

The data points in Fig. 7 are poorly centered on the curve and instead scattered. Suggesting that eqn (12) poorly predicts the water application rate. An $\mathrm{R}^{2}$ value of 0.34 is in line with this. 


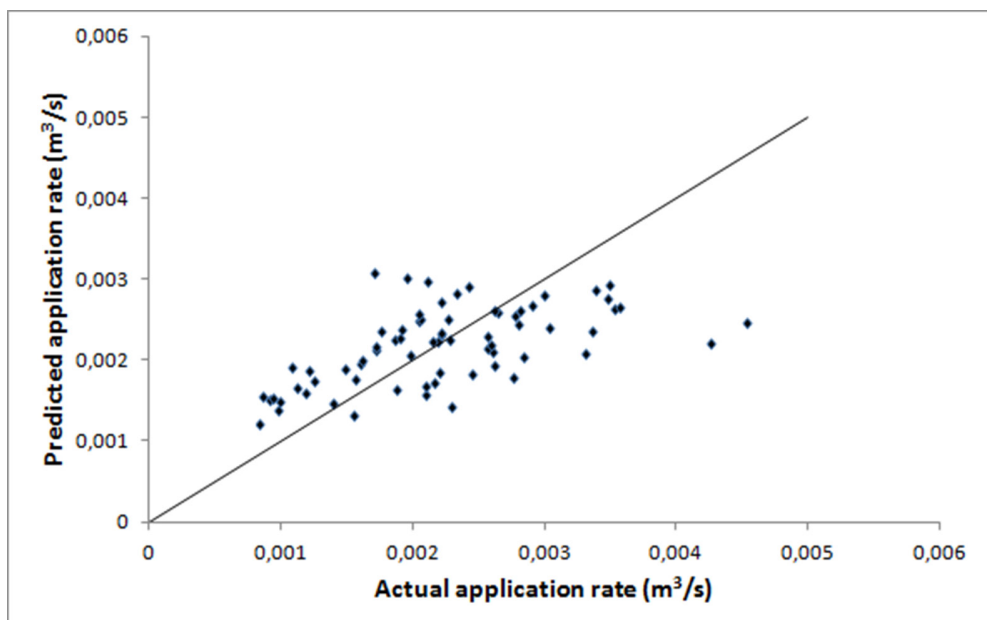

Figure 7: The application of water used during suppression - general fuel type.

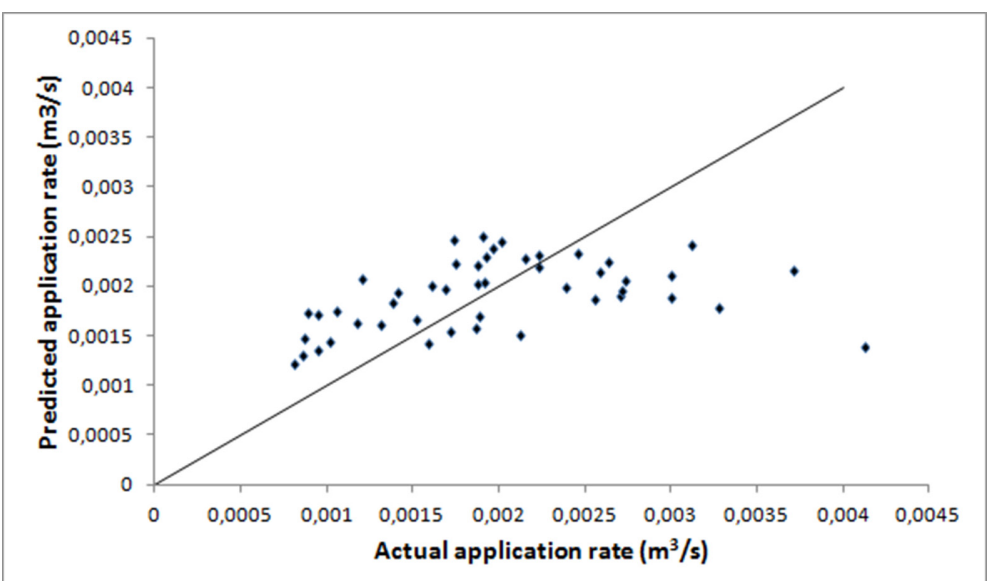

Figure 8: The application rate of water used during suppression - grass fuel type.

The best fit with respect to grass fuel type is:

$$
\dot{V}_{\text {water }}=0.001222+0.0001208 \cdot \ln \left(A_{f}\right) \quad 1 \leq A_{f} \leq 40000
$$

Equation (13) has more or less exactly the same appearance as eqn (12) but at a somewhat lower value.

Similar to the graph in Fig 7, the graph in Fig. 8 displays data points that are very poorly centered on the curve, suggesting that eqn (13) very poorly predicts the water application rate. An $\mathrm{R}^{2}$ value of 0.14 is in line with this. 


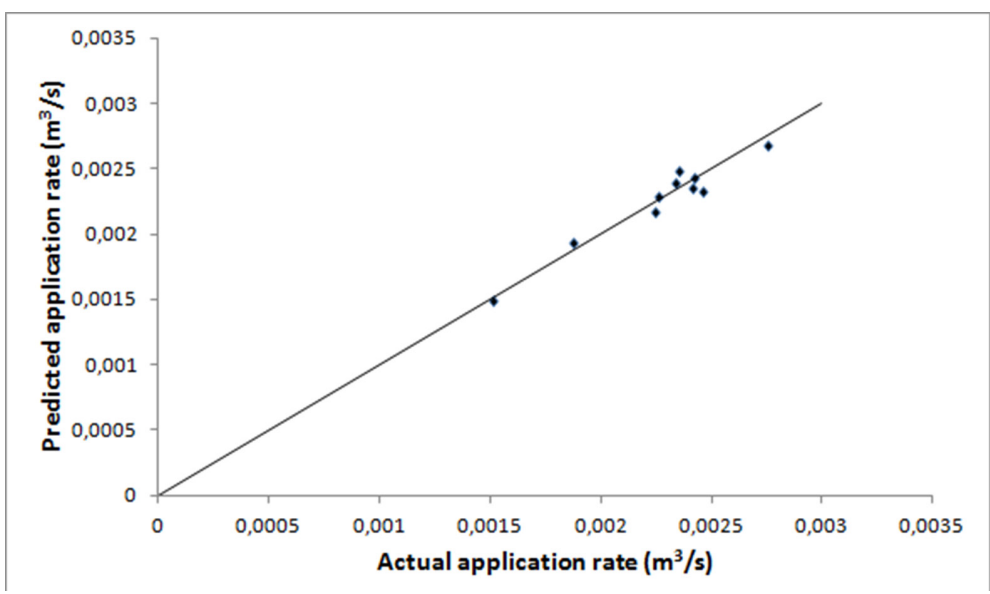

Figure 9: The application of water used during suppression - general fuel type.

5.5 The application rate of water as a function of the flame height

The best fit with respect to the general fuel type is:

$$
\dot{V}_{\text {water }}=0.002282+4.003 \cdot 10^{-5} \cdot H_{f}-\frac{3.194 \cdot 10^{-5}}{H_{f}^{2}} \quad 0.1 \leq H_{f} \leq 10
$$

Equations (14-15) describes-as many of the earlier equations-a logarithmic development. This could possibly be explained by the fact that larger flame heights are generally linked with larger, more difficult to extinguish wildfires that will take a longer time to extinguish and thus decreasing the overall application rate of water.

As can be seen in Fig. 9, the data points are nicely positioned on or very near the curve. An $\mathrm{R}^{2}$ value of 0.95 further enforces the conclusion that eqn (14) predicts the water application rate very well.

The best fit with respect to grass fuel type is:

$$
\dot{V}_{\text {water }}=0.001909 \cdot e^{-\frac{0.04683}{H_{f}}} \quad 0.1 \leq H_{f} \leq 3
$$

The data points found in the graph of Fig. 10 are fairly scattered and not centered on the curve. The poor prediction of the water application rate using eqn (15) is further enforced by an $\mathrm{R}^{2}$ value of 0.24 .

5.6 The extinguishing time as a function of the total fire area

The best fit with respect to the general fuel type is:

$$
t=1412+170 \cdot A_{f}^{0.4253} \quad 2 \leq A_{f} \leq 150000
$$

When studying eqn (16) it can be seen that the equation expresses a logarithmic development. The leveling off is due to the fact that the ratio of the active fire area to the total fire area decreases as the total fire area increases. The logarithmic development could also explain the appearance of eqn (12) i.e. also a logarithmic development. 


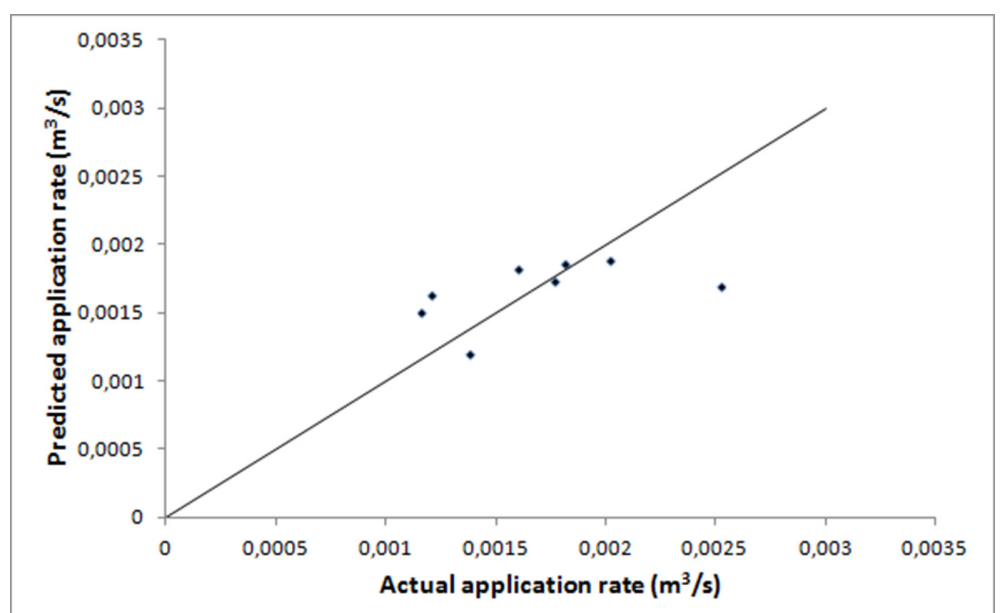

Figure 10: The application rate of water used during suppression - grass fuel type.

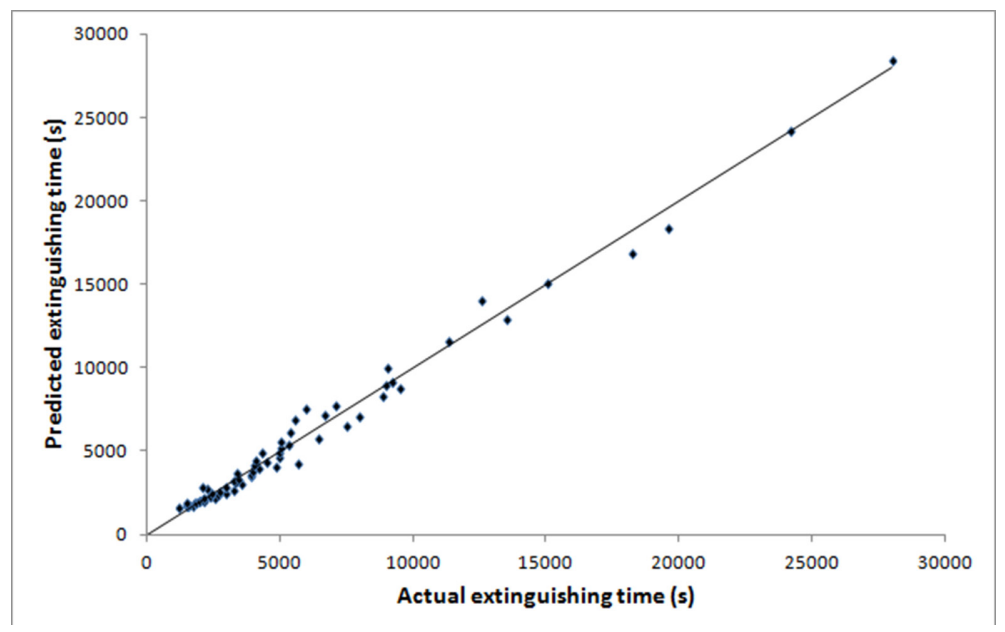

Figure 11: The extinguishing time - general fuel type.

When studying the distribution of the data points in the graph in Fig. 11, it can be seen that the data points are very nicely positioned on or very close to the curve. An $\mathrm{R}^{2}$ value of 0.99 further enforces the conclusion that eqn (16) very well predicts the extinguishing time.

The best fit with respect to grass fuel type is:

$$
t=1041 \cdot A_{f}^{0.1396} \cdot 1,00001^{A_{f}} \quad 1 \leq A_{f} \leq 30000
$$

Similar to eqn (16), eqn (17) also has a logarithmic development, but the difference in extinguishing time between the two equations increases with increasing total fire area. Wildfires in the general fuel type are thus shown to be more time-consuming than a wildfire in the grass fuel type. 


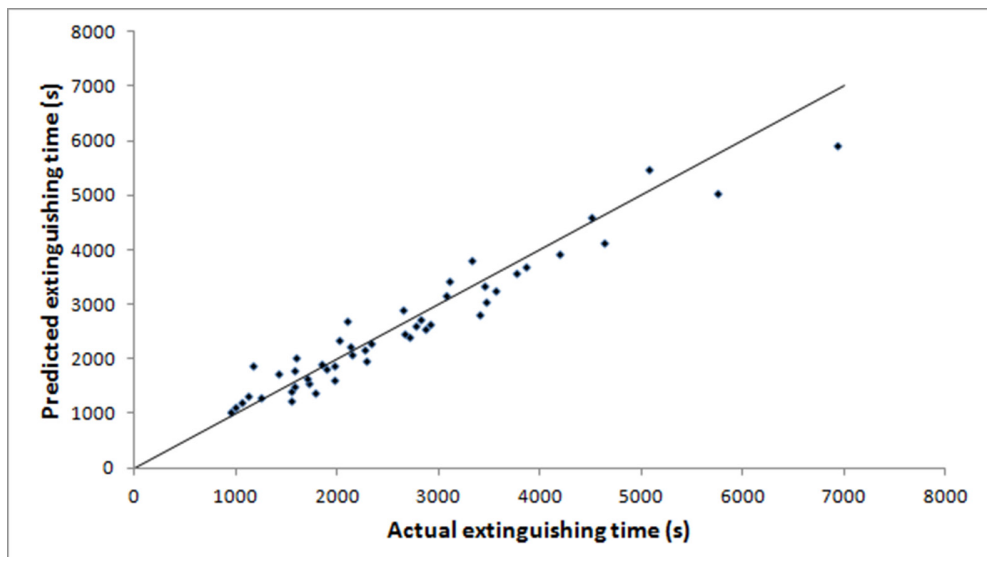

Figure 12: The extinguishing time - grass fuel type.

The distribution of the data points in the graph in Fig. 12 is very nicely positioned on or very close to the curve. Equation (17) very well predicts the extinguishing time, which is in line with an $\mathrm{R}^{2}$ value of 0.94 .

5.7 The extinguishing time as a function of the flame height

The best fit with respect to the general fuel type is:

$$
t=-400+6339 \cdot H_{f}^{0.4896} \quad 0.1 \leq H_{f} \leq 10
$$

Equation (18) displays a logarithmic development, but without any leveling off within the interval in which it is defined. Instead, it has almost a linear appearance for higher values. The lack of leveling off is encouraging as a larger flame height would imply higher heat release rate and therefore fires would be more difficult to extinguish and would have longer extinguishing times.

When studying the data points in the graph in Fig. 13, they are found to be very nicely positioned on or very close to the curve. Equation (18) therefore very well predicts the extinguishing time, which is in line with an $\mathrm{R}^{2}$ value of 0.95 .

The best fit with respect to grass fuel type is:

$$
t=2384 \cdot 0.9053^{H_{f}} \cdot H_{f}^{0.1330} \quad 0.1 \leq H_{f} \leq 5
$$

Equation (19) initially displays a logarithmic development, rapidly leveling off, but actually starts to decay somewhat for higher values. The leveling off could be due to the flashy nature of the fuel, making it easier to suppress and therefore the extinguishing time will not increase as much as for the general fuel type. The decay phase was not expected and the reason behind it is unclear.

The data points in Fig. 14 are scattered and generally not positioned especially close to the curve. A conclusion is, therefore, that eqn (19) does not predict the extinguishing time well and an $\mathrm{R}^{2}$ value of 0.43 enforces this conclusion.

\section{DISCUSSION}

The most potential relationships were found to give results that could be explained and foreseen except for one case i.e. eqn (19). The relationship was distinguished by involving the grass fuel type 


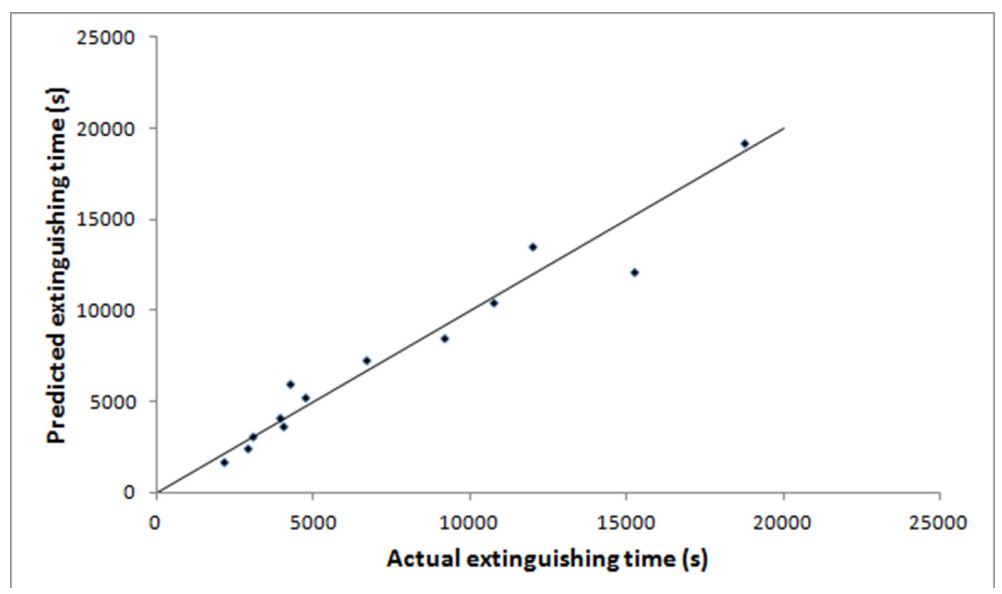

Figure 13: The extinguishing time - general fuel type.

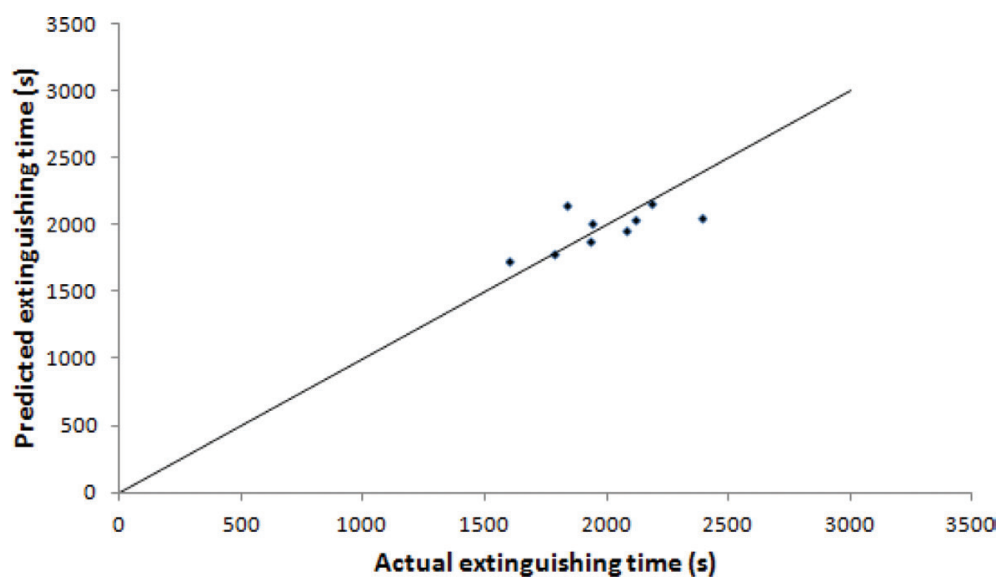

Figure 14: The extinguishing time-grass type fuel.

and the $\mathrm{R}^{2}$ value of the relationship was low and therefore the ability to predict the dependent variable was low. It could possibly be that the number of observations and the quality of the input data was low and therefore increasing the uncertainties and resulting in erroneous developments.

The relationship with the lowest $\mathrm{R}^{2}$ value was the application rate of water as a function of the total fire area. This relationship is distinguished by the large number of involved parameters, i.e. three parameters. The number of parameters and their uncertainties could add to the increased fuzziness of the output.

The relationship with the highest $\mathrm{R}^{2}$ value and highest ability to predict the dependent variable was the extinguishing time as a function of the total fire area. Apparently the extinguishing time data show a consistency which could be attributed to the fact that the start and end of the extinguishing operations are radioed in to the dispatch center and then automatically filled into the incident report by the dispatcher. The extinguishing time is not arbitrarily estimated as in the case of the flame height, total fire area, etc. 


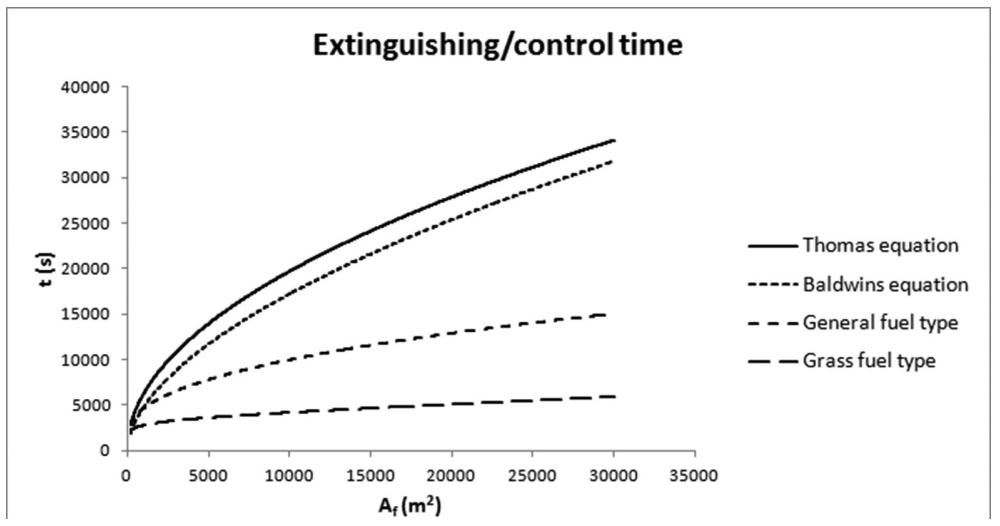

Figure 15: The control times versus the extinguishing times for both fuel types.

The control times of eqns (1) and (2) as well as the extinguishing times of eqns (16) and (17) can be found in Fig. 15. As can be seen, the control times were much longer than the extinguishing times even though the time to control a fire could be expected to be shorter than the corresponding extinguishing time. But the difference can be found in the fire area for the two different cases. In the case of building fires, the area of the fully developed fire can be expected to be fairly close to the total fire area generally being limited to the surfaces of the building in question. While in the case of a wildfire, the active fire area at a certain point of time - after the initial acceleration phase - can be expected to be considerably smaller than the total fire area.

It was found that eqns (16) and (17) could very well predict the extinguishing time. When applying eqns (16) and (17), the total fire area - i.e. the area already burnt at the time added with the active fire area is accounted for and not the active fire area. The resulting extinguishing time will therefore be longer than the duration of the water application and serve as a conservative estimate as the time will somewhat also account for mop-up, etc. and therefore reduce the impact of uncertainties in the input and output data.

Throughout the entire analysis, the relationships for the grass fuel type have generally lower $\mathrm{R}^{2}$ values and a more scattered distribution of the data points compared with the corresponding values and data points for the general fuel type. The opposite condition was expected - i.e. generally higher $\mathrm{R}^{2}$ values for the grass fuel type - as a better defined data population with respect to fuel type should improve the ability to predict the dependent variable. Why this is not the case is unclear. It could be that the number of samples for the grass fuel type should increase in order to be able to develop relationships with higher $\mathrm{R}^{2}$ values. Further investigations with respect to fuel types would be desirable. This could be attempted in a few years when the number of samples in the database has increased and therefore also the confidence in the output data, and when possibly other fuel types can be distinguished in the data material.

\section{CONCLUSIONS}

An exploratory and robust regression analysis with respect to statistical material related to the extinguishing operations of wildfires was carried out. The resulting relationships of the analysis were evaluated and discussed and some results were compared with corresponding results from building fires. The results of the analysis can be summarized as follows:

- The developed relationships had generally high $\mathrm{R}^{2}$ values and found to predict the dependent variable very well. The relationship found to have the highest ability to predict the dependent variable was the extinguishing time as a function of the total fire area. 
- The relationship with the lowest $\mathrm{R}^{2}$ value and ability to predict the dependent variable was the application rate of water as a function of the total fire area. The large number of parameters and their included uncertainties could be an explanation to the increased inability to predict the dependent variable.

- The resulting relationships could be explained and foreseen except for one case that involved the grass fuel type and the $\mathrm{R}^{2}$ value of the relationship was low and therefore the ability to predict the dependent variable was low. It could possibly be that the number and quality of samples was low, increasing the uncertainties and resulting in erroneous and inexplicable developments.

- When comparing the control times of Thomas [8]; Baldwin [9] with the extinguishing times of eqns (16) and (17), it was found that the control times were much longer than the corresponding extinguishing times even though the time to control a fire could be expected to be shorter than the extinguishing time. The difference could be found in the fire area as the area of the fully developed building fire can generally be expected to be fairly close to the total fire area. In the case of a wildfire, the active fire area could be expected to be considerably smaller than the final total fire area. When applying eqns (16) and (17) to predict the extinguishing time, one should bear in mind that the resulting output will be a conservative estimate where mop-up, etc. will also be included.

- When dividing the populations into different fuel types it was expected that for the grass fuel type the corresponding relationships would - with improved definition of data - result in higher $\mathrm{R}^{2}$ values. But the resulting relationships for the grass fuel type were found to have generally lower $\mathrm{R}^{2}$ values and more scattered data points compared with the corresponding values and data points for the general fuel type. The reason behind this is unclear, but it could be that the number of samples for the grass fuel type would have to increase in order to be able to develop relationships with higher $\mathrm{R}^{2}$ values. The issue would need to be investigated further.

\section{FURTHER WORK}

The resulting expressions in this paper should be refined further. Additional number of samples should be searched for and further analysis should be performed with respect to various types of fuels, thereby increasing the number of samples as well as differentiating the data material into several groups of fuel types. This is in order to further investigate the influence of the fuel type on the extinguishing time, etc. Data on the control time - as opposed to the total extinguishing time applied in this paper - should also be collected in order to exclude the mop up time from the data and thereby receive time periods that better reflect the actual time of water application.

\section{REFERENCES}

[1] Stechishen E., Measurement of the Effectiveness of Water as a Fire Suppressant, Forest Fire Research Institute Information report FF-X-23, Ottawa, 1970.

[2] Stechishen E. \& Little E.C., Water Application Depths Required For Extinguishment of Low Intensity Fires in Forest Fuels, Forest Fire Research Institute Information report FF-X-29, Ottawa, 1971.

[3] Loane I.T. \& Gould J.S., Project Aquarius, Aerial Suppression of Bushfires, National Bushfire Research Unit, CSIRO Division of Forest Research, Canberra, 1986.

[4] Douglas D.R., Initial Attack Standards, Research working group no 6, Newsletter no 4, July 1973.

[5] Andrews P.L. \& Rothermel R.C., Charts for Interpreting Wildland Fire Behaviour Characteristics, USDA Forest Service, Intermountain Forest and Range Experiment Station General Technical Report INT-131, Ogden, 1982.

[6] Alexander M.E., Fire Behaviour as a Factor in Forest and Rural Fire Suppression, New Zealand Fire Service Commission \& The National Rural Fire Authority Forest research bulletin no 197 Forest and rural fire scientific and technical series report no 5, Wellington, 2000. 
[7] Hansen R, Estimating the amount of water required to extinguish wildfires under different conditions and in various fuel types. International Journal of Wildland Fire, 21, 525-536, 2012. doi: http://dx.doi.org/10.1071/wf11022

[8] Thomas P.H., A Note on the Extinguishment of Very Large Fires, Fire Research Note 245, Borehamwood, England, 1957.

[9] Baldwin R., The Use of water in the Extinguishment of Fires by Brigades, Fire Research Note 803, Borehamwood, England, 1970.

[10] Lundqvist M., Electronic correspondence with Marie Lundqvist, MSB, Sweden 2011-12-21, 2011.

[11] Malmqvist M., Electronic correspondence with Mikael Malmqvist, MSB, Sweden 2012-06-29, 2012.

[12] Mosteller F. \& Tukey J., Data Analysis and Regression, a Second Course in Statistics, Pearson, 1977.

[13] Elliott A.C. \& Woodward W.A., Statistical Analysis Quick Reference Guidebook, Sage Publications Inc.: Thousand Oaks, 2007. 